\title{
Frakturen im höheren Lebensalter - eine Herausforderung für Prävention und Gesundheitsförderung - Ergebnisse der KORA-Frakturstudie Augsburg
}
M. Wildner ${ }^{1,2}$
A. Döring ${ }^{3}$
C. Meisinger ${ }^{3}$
D. E. Clark $^{4}$

\author{
Fractures in the Elderly - a Challenge for Prevention and Health Promotion - \\ Results from the KORA Fracture Study Augsburg
}

\section{Zusammenfassung}

Frakturen der Extremitätenknochen sind häufig und können - abhängig von Lebensalter und Lokalisation - auch Ursachen eines Verlustes der Fähigkeit zur unabhängigen Lebensführung sein. Sie betreffen beide Geschlechter zu unterschiedlichen Zeitpunkten und in unterschiedlicher Gestalt. Die Basis-Epidemiologie dieser Erkrankungen bzw. Unfallfolgen und Aussagen zu den mittelfristigen gesundheitlichen Folgen insbesondere bei Fragilitätsfrakturen sind im Rahmen der Augsburger KORA-Frakturstudie 1998, eines Anschlussprojektes für ältere Teilnehmer des dritten MONICA-Augsburg-Surveys S3 (1994/95) und begleitender Forschungen für Deutschland in den vergangenen Jahren erarbeitet worden. Ergebnisse einer Validierungsstudie belegen die Gültigkeit fragebogengestützter Selbstauskünfte zu Frakturen und ihren Lokalisationen. Es konnte gezeigt werden, dass Alter, Geschlecht, Zeit seit der Fraktur und Beeinträchtigung der Gehfähigkeit unter einer Vielzahl von Einflussgrößen die größte prädiktive Wertigkeit aufweisen. Einschränkungen der körperlichen Funktionen sind insbesondere in den ersten beiden Jahren nach einer Fraktur nachweisbar. Nach fünf Jahren entsprechen die funktionalen Werte bei den Überlebenden denen von Kontrollpersonen gleichen Alters. Diese bevölkerungsrepräsentativen und validiert vorliegenden Daten unterstützen modellgestützte Aussagen zu einer kosteneffektiven bevölkerungsbezogenen Strategie der Prävention und Gesundheitsförderung. Eine alters- und geschlechtsspezifische Herangehensweise ist unverzichtbar (Gender Mainstreaming).

\section{Abstract}

Fractures of the appendicular bones are frequent and can cause a loss of the ability for independent living, depending on age and fracture localisation. They affect both sexes at different times and in different ways. The basic epidemiology for Germany of this injury resp. disease and knowledge regarding mediumterm health-related consequences has been provided by work done in the context of the KORA Augsburg Fracture Study 1998, a follow-up of older participants of the third MONICA Augsburg survey S3 (1994/95) and through further research in the past years. Results of a validation study support the value of questionnaire-based patients recall of fractures and their location. It could be shown that age, gender, time since fracture and impaired walking have the greatest predictive value among a number of factors. Limitations of physical function are apparent especially during the first two years after a fragility fracture. After five years functional capacity of survivors equals the functional capacity of control persons of the same age. These population-representative and validated data support model-based cost-effective strategies of population-orientated disease prevention and health promotion. An age and gender-specific approach (gender mainstreaming) is mandatory.

\section{Key words}

Epidemiology $\cdot$ fractures $\cdot$ prevention $\cdot$ health promotion

\section{Schlüsselwörter}

Epidemiologie · Frakturen · Prävention · Gesundheitsförderung

Institutsangaben

${ }^{1}$ Bayerisches Landesamt für Gesundheit und Lebensmittelsicherheit, Oberschleißheim

${ }^{2}$ Ludwig-Maximilians-Universität München, Institut für Medizinische Informationsverarbeitung, Biometrie und Epidemiologie, München

${ }^{3}$ GSF-Forschungszentrum für Umwelt und Gesundheit, Institut für Epidemiologie, Neuherberg

${ }^{4}$ Harvard Injury Control Research Center, Boston

Korrespondenzadresse

Priv.-Doz. Dr. Manfred Wildner • Bayerisches Landesamt für Gesundheit und Lebensmittelsicherheit .

Veterinärstraße 2·87654 Oberschleißheim ·E-mail: manfred.wildner@lgl.bayern.de

Bibliografie

Gesundheitswesen 2005; 67 Sonderheft 1: S180-S186 @ Georg Thieme Verlag KG Stuttgart • New York DOI $10.1055 / \mathrm{s}-2005-858262$

ISSN 0949-7013 


\section{Einleitung}

Von der Weltgesundheitsorganisation wurde das erste Jahrzehnt des 21. Jahrhunderts zur „Bone and Joint Decade“ erklärt (www.boneandjointdecade.org/). Knochenbrüche sind in allen Lebensaltern anzutreffen - abhängig vom Geschlecht mit unterschiedlichen Häufigkeitsgipfeln - und betreffen Männer und Frauen gleichermaßen. Osteoporose, eine chronisch-progrediente Erkrankung des Skeletts, ist in diesem Zusammenhang als wichtiges Gesundheitsproblem in vielen Industrieländern erkannt. Etwa jede zweite Frau wird eine osteoporosebedingte Fragilitätsfraktur im Laufe ihres Lebens erleiden, aber nur etwa jeder zehnte Mann. Seit langem ist bekannt, dass diese Fragilitätsfrakturen insbesondere die Wirbelkörper, den distalen Radius (handgelenksnaher Unterarm), den proximalen Humerus (schultergelenksnaher Oberarm), das proximale Femur (hüftnaher Oberschenkel), die proximale Tibia und Fibula (kniegelenksnaher Unterschenkel) und die Malleolen (fußgelenksnaher Unterschenkel) betreffen [1]. Unter den genannten Frakturen ist die proximale Femurfraktur die folgenschwerste Form.

Diese Situation hat Anstrengungen zur Ursachenforschung, Prävention und epidemiologischen Überwachung ausgelöst [2]. Maßnahmen zur Prävention der proximalen Femurfraktur sind Bestandteil nationaler und regionaler Gesundheitsziele. Die Weltgesundheitsorganisation (WHO) betrachtet Osteoporose als globales Problem und nennt als vordringliche Forschungsschwerpunkte Untersuchungen zur Frakturepidemiologie und zu kostengünstigen bzw. kosteneffizienten Präventionsstrategien [3]. Untersuchungen zur Häufigkeit von Wirbelkörperfrakturen wurden in der Vergangenheit in europäischer Kooperation auch in Deutschland durchgeführt (EVOS-Studie, [4]).

Übergeordnetes Ziel der vorliegenden Arbeit ist es, den epidemiologischen Wissensstand zu Extremitätenfrakturen in Deutschland durch eine zusammenfassende Darstellung der eigenen Forschungen im Rahmen der Augsburger KORA-Frakturstudie zu verbessern und exemplarisch für Fragilitätsfrakturen
Anregungen für die Entwicklung und Bewertung bevölkerungsmedizinischer Präventionsempfehlungen zu geben.

\section{Methodik}

Die nachfolgend dargestellten Arbeiten im Rahmen der KORAFrakturstudie sind Teil eines umfassenderen Forschungsansatzes, welcher sich über einen Zeitraum von etwa 15 Jahren erstreckt. Diese Forschungen begannen nach der deutschen Wiedervereinigung mit Arbeiten zur Basisepidemiologie der proximalen Femurfraktur an Datensätzen der DDR an der Abteilung Epidemiologie nicht übertragbarer Krankheiten am Robert-Koch-Institut in Berlin (damals noch Bundesgesundheitsamt) und setzten sich fort mit Arbeiten zur Erforschung eines säkularen Trends bei proximalen Femurfrakturen, zur Basisepidemiologie von Extremitätenfrakturen und zu funktionellen gesundheitlichen und gesundheitsökonomischen Folgen im Rahmen der Augsburger Frakturstudie, zum evidenzbasierten Risiko-Assessment sowie zur entscheidungsanalytisch begründeten Formulierung von Präventionsempfehlungen.

Zur Bearbeitung der verschiedenen Fragestellungen wurden verschiedene Datenkörper herangezogen. Tab. 1 gibt eine Übersicht über die Zuordnung zu den Fragestellungen. Die verwendeten Datenkörper wurden sorgfältig ausgewählt und teilweise mit eigens berechneten Korrekturfaktoren aufbereitet. Zusätzlich wurden Qualitätsvergleiche aufgrund der ICD-Verschlüsselung vorgenommen und die Qualität auch im zeitlichen Verlauf beurteilt. Für die zeitlichen und regionalen Vergleiche wurde eine Standardisierung der Daten nach Alter und Geschlecht durchgeführt. In der KORA-Frakturstudie wurden auf Basis einer gezielten Auswertung des MONICA-Survey S3 (1994/95) zusätzlich im Jahr 1998 eigene Daten prospektiv erhoben und validiert. Eine Beschreibung der verwendeten Datenkörper und Methoden wurde publiziert [5 - 8]. Die im Rahmen des Surveys S3 erzielten Forschungsergebnisse bilden den Schwerpunkt der vorliegenden Arbeit.

Tab. 1 Übersicht und Zuordnung der Datenkörper zu den spezifischen Fragestellungen

$\begin{array}{ll}\text { Datenkörper } & \begin{array}{l}\text { Alters- und geschlechts- säkularer Trend gesundheitliche kosteneffektive Präven- } \\ \text { spezifische Inzidenz- } \\ \text { proximaler Folgen und ihre tionsmaßnahmen Prävalenzraten Femurfrakturen Prädiktoren }\end{array}\end{array}$

\begin{tabular}{|c|c|c|c|c|}
\hline $\begin{array}{l}\text { Krankenhausdiagnosestatistik der GKV nach § } 79 \text { SGB IV, alte Bun- } \\
\text { desländer }{ }^{1}\end{array}$ & $\mathrm{x}$ & & & \\
\hline Krankenhausdiagnosestatistik der DDR ${ }^{1}$ & $x$ & $x$ & & $x$ \\
\hline $\begin{array}{l}\text { Krankenhausdiagnosestatistik der Krankenhausstatistikverordnung } \\
\text { vom } 10.4 .1990^{2}\end{array}$ & $\mathrm{x}$ & $x$ & & $\mathrm{x}$ \\
\hline Diagnose- und Therapieindex (DTI), I + G Gesundheitsforschung² & $\mathrm{x}$ & $x$ & & \\
\hline National Health and Nutrition Examination Survey (NHANES III, USA) ${ }^{3}$ & $\mathrm{x}$ & & & $x$ \\
\hline MONICA-Survey 1994/94 (S3) & $x$ & & & \\
\hline KORA-Frakturstudie ${ }^{4}$ & & & $x$ & $x$ \\
\hline
\end{tabular}

${ }^{1}$ Die Auswertung am Robert-Koch-Institut Berlin wurde unterstützt durch ein Stipendium der Dr.-Liesel-Keinath-Stiftung des Stifterverbandes der Deutschen Wissenschaft.

2 Die Auswertung erfolgte im Rahmen des für das BMG durchgeführten Pilotprojektes „Operationshäufigkeiten in Deutschland“.

3 Die Auswertung wurde unterstützt durch das „MSD Sharp \& Dohme“-Förderstipendium Osteoporose 1999.

4 In der Studie, die durch das BMFT im Rahmen der 2. Förderphase des Bayerischen Forschungsverbundes Public Health - Öffentliche Gesundheit gefördert wurde, wurden eigene Daten erhoben. 
Neben Standardverfahren der deskriptiven Statistik kamen multiple logistische Regressionsmodelle bei dichotomer Zielvariable sowie multiple lineare Regressionsmodelle bei kontinuierlicher Zielvariable zur Anwendung, in den vorbereitenden bzw. fortführenden Forschungen multivariable Poisson-Regression zur Analyse eines säkularen Trends, Methoden der kleinräumigen Datenanalyse (Small Area Analysis) bei der Analyse regionaler Unterschiede sowie die Methode der Entscheidungsanalyse für die Formulierung von Interventionsempfehlungen auf Basis der epidemiologischen und gesundheitsökonomischen Daten. Zufallseinflüsse wurden durch Angabe von 95\%-Vertrauensbereichen berücksichtigt, soweit es sich nicht um Vollerhebungen handelte.

\section{Ergebnisse}

\section{Alters- und geschlechtsspezifische Inzidenz- bzw. Prävalenzraten}

Über den dritten MONICA-Augsburg-Survey S3 wurden von 4854 Personen im Alter von 25 bis 74 Jahren (2404 Männer, 2450 Frauen) Daten zu Knochenbrüchen (Extremitätenfrakturen) aus Eigenangaben erhoben. Bei 146 Fällen mit Eigenangabe einer Fraktur waren diese Angaben bezüglich Validität und Reliabilität geprüft worden (Anschreiben der Hausärzte) [6]. Der Kappa-Wert lag dabei für die Reliabilität der Probandenangaben zu den genannten Zeitpunkten bei 0,80-0,89 und für die Validität bei 0,77-0,89. Die Erhebung von Probandenangaben zu Frakturen stellt somit eine zuverlässige Methode dar, um Daten für epidemiologische Fragestellungen zu gewinnen (Abb.1).

Die höchsten frakturspezifischen Inzidenzraten fanden sich bei Frauen in der Altersgruppe 65 - 74 Jahre für Unterarm- und Unterschenkelfrakturen (982,1/100000 Personenjahre bzw. 535,7/100 000 Personenjahre). Bei Männern lagen die höchsten Inzidenzraten bei 416,0/100000 Personenjahre bzw. bei 370,2/100 000 Personenjahre für Hand- bzw. Unterschenkelfrakturen, jeweils in der Altersgruppe 15-24 Jahre. Sowohl bei Männern als auch bei Frauen mit Unterarm- bzw. Unterschenkelfrakturen handelt es sich zum größten Teil um Handgelenksbzw. Malleolarfrakturen. Durch einen Sturz wurden 43\% (Frauen 59\%, Männer 33\%), durch äußere Gewalteinwirkung 40\%
(Männer 47\%, Frauen 29\%) und durch sportliche Aktivitäten 15\% (Männer 18\%, Frauen 10\%) aller Frakturen verursacht [7]. Die Sturzprävention bei älteren Menschen ist somit für die $\mathrm{Zu}$ kunft ein wichtiges Anliegen der öffentlichen Gesundheit.

\section{Gesundheitliche Folgen und ihre Prädiktoren}

Gesundheitliche Auswirkungen der Frakturen betreffen den funktionellen Gesundheitszustand in verschiedenen Dimensionen sowie die frühe und späte Morbidität bzw. Letalität. Frühe Komplikationen der operativen und nichtoperativen Frakturbehandlung sind in der Literatur hinreichend behandelt. Eigenen Berechnungen zufolge liegt in Deutschland die krankenhausbezogene Letalität nach proximalen Femurfrakturen in der ersten Lebenshälfte bei unter einem Prozent und steigt für ältere Menschen bis auf $12 \%$ und höher an. Dabei existieren erhebliche regionale Unterschiede, welche zumindest für die DDR am ehesten durch die Qualität der medizinischen Versorgung erklärbar waren [5, 9]. Über die unmittelbaren medizinischen Auswirkungen hinaus sind die Folgen von Fragilitätsfrakturen im höheren Lebensalter nur unzureichend untersucht. Im Rahmen der KORA-Frakturstudie konnten 1999 insgesamt 146 Personen im Alter von 58-78 Jahren mit Frakturanamnese sowie 311 nach Alter und Geschlecht zugeordnete Kontrollpersonen nachuntersucht werden („nested case-cohort design“). Dabei wurden als patientenzentrierte Befragungsinstrumente zur Messung der generischen Lebensqualität die Medical Outcomes Study Short Form 36 (SF 36) eingesetzt und für die erkrankungsspezifische funktionale Gesundheit der Functional Disability Index (FDI, Abb. 2).

Der Effekt der Fraktur und anderer Variablen auf die Fähigkeit zur unabhängigen Lebensführung wurde über ein lineares Regressionsmodell mit Backward Elimination der potenziellen Einflussgrößen untersucht, das Modell ist in Tab. 2 wiedergegeben. Zu erklärende Variable war der Functional Disability Index (FDI), der Summenscore des Health Assessment Questionnaires (HAQ) mit Fragen zu Aktivitäten und komplexeren instrumentellen Aktivitäten des täglichen Lebens. In das multivariabel adjustierte Modell wurden neben verschiedenen Einflussgrößen Design-Variablen für Frakturen der langen Röhrenknochen der oberen und unteren Extremität aufgenommen.

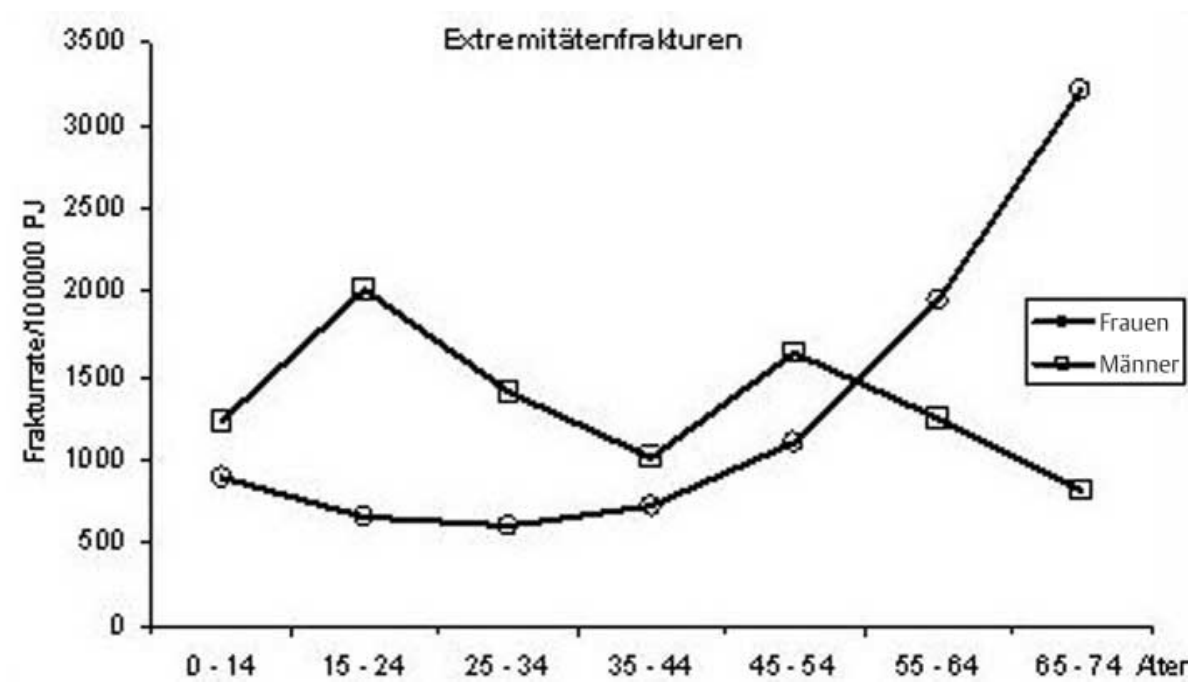

Abb. 1 Alters- und geschlechtsspezifische Frakturraten (Alter bei Fraktur), alle Frakturen (MONICA-Survey 1994/95 [S3]). 


\begin{tabular}{|c|c|c|}
\hline $\begin{array}{l}\frown \text { Bestmöglich } \\
\text { ¿- Obers cherkelfraturen }\end{array}$ & $\begin{array}{l}\text { —-Kont ollen } \\
\text { *-Unter armfraturen }\end{array}$ & $\therefore$ Alle F rakturen \\
\hline
\end{tabular}

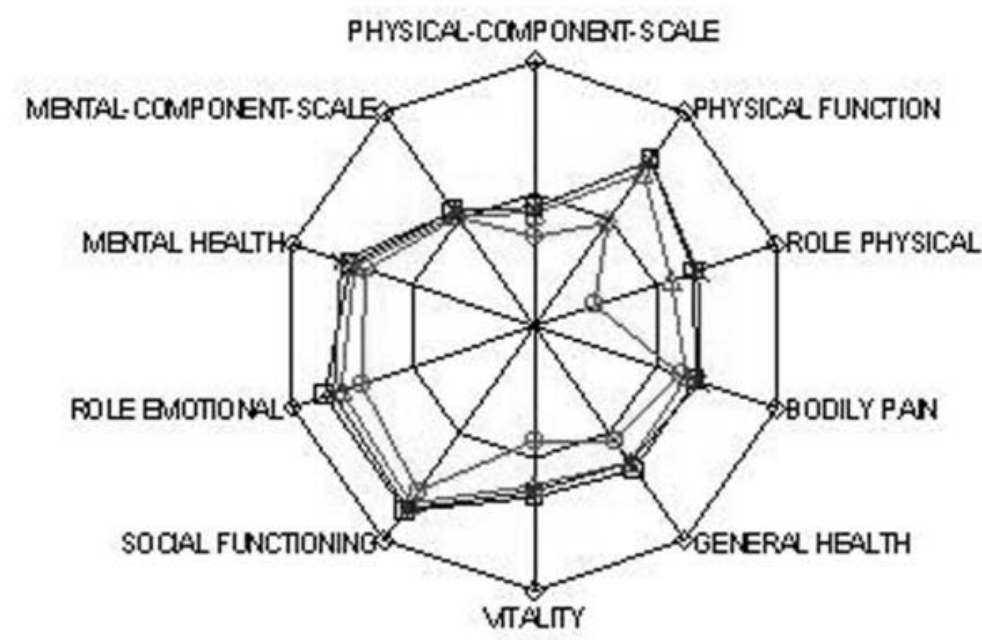

Die subjektive globale Selbsteinschätzung der Gesundheit korreliert nur mäßig mit dem Summenscore FDI der körperlichen Funktionsfähigkeit $(r=0,48)$. Das Obermodell 2 der multiplen linearen Regression mit den Forced-in-Variablen Alter, Geschlecht und Status als Fall oder Kontrolle und weiteren statistisch signifikanten Einflussgrößen erklärte über 50\% der beobachteten Varianz der körperlichen Funktion, gemessen mit dem FDI. Bei einer Analyse des erklärenden Einflusses der Subskalen des HAQ war der größte Effekt in Zusammenhang mit Gehen, Erreichen (Arm) und Schmerz zu beobachten. Andere bedeutsame Einflussgrößen wa-
Abb. 2 Ergebnisse der Augsburger KORAFrakturstudie 1999. a: Körperliche Funktionsfähigkeit nach den Dimensionen des allgemeinen Gesundheitszustandes nach der Medical Outcomes Study Short Form 36 (SF-36) als Langzeitergebnis nach Extremitätenfrakturen. Die Distanz zwischen Zentrum und äußerem Kreis (bestmögliche Funktionsmessung) entspricht Funktionswerten von $0-100 \%$. Die Physical und die Mental Component Scale sind zusammenfassende Indizes der jeweiligen Dimensionen. b: Spezifische körperliche Funktionsfähigkeit nach den Dimensionen des Health Assessment Questionnaire (HAQ) als Langzeitergebnis nach Extremitätenfrakturen. Die Distanz zwischen Zentrum und äußerem Kreis (bestmögliche Funktionsmessung) entspricht Punktwerten von $0-3$. Der Functional Disability Index fasst die verschiedenen Dimensionen zusammen. Es wird deutlich, dass gegenüber den Kontrollpersonen insbesondere bei proximalen $\mathrm{Fe}$ murfrakturen noch über mehrere Jahre funktionale Defizite nachweisbar sind. ren eine Schlaganfallanamnese oder andere neurologische Störungen, Depressionen/Gemütsstörungen, eine Oberschenkelfraktur, die allgemeine Komorbidität, die seit der Fraktur vergangene Zeit, Übergewicht sowie die Anzahl und Qualität sozialer Kontakte. Der größte Beitrag zur erklärten Varianz kam dabei von der An- oder Abwesenheit von Gehstörungen.

Ein reduziertes Untermodell, welches außer den Forced-in-Variablen nur die Eigenangabe Gehstörungen enthielt, war in der Lage, noch immer $44 \%$ der beobachteten Varianz zu erklären. 


\begin{tabular}{|c|c|c|c|c|c|}
\hline \multirow[t]{2}{*}{ Modell } & \multirow[t]{2}{*}{ Variable } & \multirow{2}{*}{$\begin{array}{l}\text { nicht stand. } \\
\text { Koeffizient Beta }\end{array}$} & \multirow[t]{2}{*}{$p$-Wert } & \multicolumn{2}{|c|}{ 95\% Konfidenz-Intervall für Beta } \\
\hline & & & & Untergrenze & Obergrenze \\
\hline 1 & Konstante & $-0,224$ & 0,118 & $-0,505$ & 0,057 \\
\hline \multirow[t]{11}{*}{$R^{2} 0.51$} & Geschlecht & 9,599E-02 & 0,026 & 0,011 & 0,181 \\
\hline & Alter $^{5}$ & $4,868 \mathrm{E}-02$ & 0,254 & $-0,035$ & 0,132 \\
\hline & Gehstörung & $1,133 \mathrm{E}-02$ & 0,799 & $-0,076$ & 0,099 \\
\hline & SF Comorbid ${ }^{1}$ & 0,809 & $<0,001$ & 0,698 & 0,921 \\
\hline & Zeit nach Fraktur $^{1}$ & 0,120 & $<0,001$ & 0,060 & 0,181 \\
\hline & Oberschenkelfraktur & 0,600 & 0,014 & 0,123 & 1,077 \\
\hline & Schlaganfall (neurol. Stör.) & 0,331 & 0,040 & 0,016 & 0,647 \\
\hline & Status Fall/Kontrolle & 0,316 & $<0,001$ & 0,141 & 0,491 \\
\hline & Social Network Index & $-5,476 \mathrm{E}-02$ & 0,028 & $-0,103$ & $-0,006$ \\
\hline & $\mathrm{BMI}^{1}$ & 0,107 & 0,009 & 0,027 & 0,187 \\
\hline & Gemütserkr./ Depression & 0,143 & 0,034 & 0,011 & 0,274 \\
\hline 2 & Konstante & $-0,194$ & 0,030 & $-0,368$ & $-0,019$ \\
\hline \multirow[t]{4}{*}{$R^{2} 0.44$} & Geschlecht & 0,157 & $<0,001$ & 0,071 & 0,242 \\
\hline & Alter ${ }^{1}$ & 0,101 & 0,023 & 0,014 & 0,187 \\
\hline & Status Fall/Kontrolle & 3,073E-02 & 0,495 & $-0,058$ & 0,119 \\
\hline & Gehstörung & 0,942 & $<0,001$ & 0,835 & 1,050 \\
\hline
\end{tabular}

Tab.2 Modellierung der Einflüsse auf die unabhängige Lebensführung nach Frakturen im höheren Lebensalter im Rahmen der KORA-Frakturstudie Augsburg 1999 (Anschlussuntersuchung älterer Teilnehmer des MONICA-Surveys 1994/95 [S3]) mittels linearer Regressionsmodelle: Obermodell 1 und reduziertes Untermodell 2 mit zugehöriger erklärter Varianz $\left(R^{2}\right)$ und Koeffizienten; abhängige Variable: Functional Disability Index (FDI)

${ }^{1}$ zur vorgenommenen Kategorisierung siehe (8)

Dieser erklärte Varianzanteil war robust gegenüber dem Einoder Ausschluss hüftnaher Femurfrakturen, war unbeeinflusst von einem Interaktionsterm zwischen Gehstörung und Frakturen und blieb hoch auch nach Austausch der abhängigen Variablen FDI gegen Dimensionen der körperlichen Funktion aus dem SF-36-Fragebogen.

Es zeigte sich, dass Extremitätenfrakturen noch nach mehreren Jahren zu messbaren Beeinträchtigungen der funktionalen Gesundheit bei Personen ab dem 65. Lebensjahr führten, insbesondere bei Beteiligung des Oberschenkelknochens [8]. Die wesentlichen gesundheitlichen Folgen bei Überlebenden von Frakturen der oberen oder unteren Extremität betrafen die körperliche Funktion, und hier langfristig vor allem die Funktionen, welche Gehen, Aktivitäten außer Haus und die Körperpflege betreffen. Die Defizite waren in den ersten beiden Jahren nach der Fraktur am stärksten ausgeprägt, nach fünf Jahren hatte sich die funktionale Gesundheit den Kontrollen wieder angeglichen.

In ihrem Selbstverständnis verbanden über zwei Drittel der Befragten den Begriff „unabhängige Lebensführung“ mit „gesund sein“ und „alles tun können“. Nur wenige der Befragten nannten finanzielle Aspekte an erster Stelle. Krankheit wurde als größte Bedrohung einer unabhängigen Lebensführung genannt, gelegentlich auch ungünstige politische, soziale und finanzielle Entwicklungen. Nur ein Drittel der Befragten gab an, in der Fähigkeit zur unabhängigen Lebensführung nicht bedroht zu sein. Weniger als ein Fünftel der Befragten fühlte sich hinsichtlich Themen wie Anpassung des Wohnraumes, altengerechtes Wohnen und medizinische Hilfsmittel gut informiert $(13,1 \%, 15,8 \%$ and $16,2 \%$ ). Aspekte eines „Rechtes auf Gesundheit“ wurden im Rahmen der Augsburger Frakturstudie weiter verfolgt und mündeten in die Entwicklung eines Fragebogeninstrumentes zu Assessment-Zwecken [10].

\section{Diskussion}

\section{Inzidenz, Prävalenz und gesundheitliche Folgen}

In der vorangegangenen Zusammenfassung wurden Arbeitsergebnisse zur Häufigkeit von Knochenbrüchen aus dem Survey S3 vorgestellt sowie zu den gesundheitlichen Folgen von Fragilitätsfrakturen im Alter aus dem Survey S4 (Letalität, funktionelle Einschränkungen bei Aktivitäten des täglichen Lebens). Die vom Patienten als solche erlebte Dramatik einer Fraktur mit akutem Schmerz und zumeist auch Bewegungsunfähigkeit des betroffenen Gliedmaßenabschnittes macht in aller Regel einen Arztbesuch notwendig. Eigenangaben zu Knochenbrüchen können somit bei entsprechender Dokumentation durch den behandelnden Arzt validiert werden, wie dies von uns gemacht wurde [6, 7]. Auch wenn sich durch Anamnese und klinisches Bild häufig die richtige Diagnose einer Fraktur vermuten lässt, wird sie doch in der ganz überwiegenden Zahl der Fälle aufgrund des Röntgenbildes gestellt. Nur in seltenen Fällen macht eine sog. „schleichende Fraktur“ weitere diagnostische Maßnahmen wie die Kernspintomographie oder die Skelettszintigraphie notwendig oder entgeht als eingestauchte, belastungsstabile Fraktur der Diagnose.

Aussagen zu Risikofaktoren, Mortalität, verbleibender Morbidität und Präventionsmaßnahmen machten eine weiterführende Beobachtung notwendig. Informationen zum Gesundheitsstatus wurden in der KORA-Frakturstudie mit international breit eingesetzten, für Deutschland validierten Fragebogeninstrumenten gewonnen und können als zuverlässig eingeschätzt werden [11 - 13]. Lebensalter, männliches Geschlecht, (Art der) Fraktur und Schwierigkeiten beim Gehen erscheinen als signifikante und wichtige Prädiktoren der funktionalen Gesundheit nach Fragilitätsfrakturen.

Zwei Drittel der Befragten gaben darüber hinaus an, in der Fähigkeit zur unabhängigen Lebensführung bedroht zu sein. Nur eine Minderheit der Befragten fühlte sich hinsichtlich Themen wie Anpassung des Wohnraumes, altengerechtes Wohnen und medi- 
zinische Hilfsmittel gut informiert. Es wird dabei deutlich, dass die bestehenden Systeme der medizinischen Versorgung subjektiv als wichtig empfundene und für die Prognose bedeutsame Aspekte der tertiären Rehabilitation ungenügend abdecken. Die identifizierten Defizite haben zum einen hinsichtlich der Behandlungsqualität Bedeutung, zum anderen hinsichtlich der Responsiveness der Systeme im Sinne von Patientenrechten. Weiterführende Arbeiten zum innovativen Assessment wichtiger Aspekte des Rechtes auf Gesundheit wurden publiziert [10].

\section{Kosteneffektive Maßnahmen der Prävention und Gesundheitsförderung}

Auf Basis der berichteten epidemiologischen Daten wird deutlich, dass sich das Frakturgeschehen bei Frauen und Männern auf unterschiedliche Lebensalter und unterschiedliche Frakturumstände bezieht [7]. Daraus leitet sich die Forderung nach alters- und zielgruppenspezifischen Präventions- bzw. Gesundheitsförderungsmaßnahmen ab. Grundsätzlich sind insbesondere bei Fragilitätsfrakturen verschiedene Maßnahmen der Ressourcenstärkung (Gesundheitsförderung) und der Belastungssenkung (Prävention) zu diskutieren bzw. zu evaluieren. Der Gesundheitsförderung zuzurechnen wären z. B. eine Erhöhung der Peak Bone Mass in jüngeren Jahren durch entsprechende Ernährungs- und Bewegungsangebote oder die Förderung der motorischen Fähigkeiten im höheren Alter. Präventionsmaßnahmen betreffen neben der allgemeinen medizinischen Versorgung insbesondere einen Erhalt des Knochenmineralgehaltes, welcher über die Knochendichte (BMD) an standardisierten Messorten abgebildet werden kann.

Präventionsempfehlungen bzgl. Osteoporose und proximalen Femurfrakturen stehen in enger Verbindung zu diagnostischen Maßnahmen, insbesondere zur Indikationsstellung der Osteodensitometrie. Folgerichtig wurde die Rolle der Osteodensitometrie im Rahmen der Primär-, Sekundär- und Tertiärprävention zum Gegenstand eines deutschen Health-Technology-Assessment(HTA)-Berichtes [14]. Der deutsche HTA-Bericht kommt ebenso wie wir zu dem Schluss, dass die Indikationsstellung zur Osteodensitometrie nur bei Hochrisikogruppen als selektives Screening zu stellen ist und die Entwicklung eines validierten Fragebogen-Screening-Instrumentes zur Bestimmung des Frakturrisikos aufgrund von Risikofaktoren dringend zu fordern ist [5,14]. Andere Arbeitsgruppen schließen sich dieser Empfehlung an $[15-17]$.

Die Ergebnisse der Entscheidungsanalyse hängen natürlich von den eingegebenen bzw. einzugebenden Voraussetzungen ab (Kosten- und Effektivitätsschätzungen, alters- und geschlechtsspezifische Frakturrisiken, alters- und geschlechtsspezifische Osteoporoserisiken, Korrekturfaktoren für deren Berechnungen u.a. m.), von den Unrelated Costs in der gewonnenen Lebenszeit, den indirekten Kosten z.B. durch pflegende Angehörige und wären bei einer Anwendung auf deutsche Verhältnisse hinsichtlich der allokationstheoretischen Begründung innerhalb eines Systems fixer Budgets weiter zu diskutieren. Trotzdem zeigen die Analysen in ihrer Gesamtheit, dass eine rationale Bewertung konkurrierender Strategien zur medikamentösen Prävention proximaler Femurfrakturen möglich ist. Dabei kommt dem Assessment des individuellen A-priori-Frakturrisikos für die Entscheidungsfindung eine besonders wichtige Bedeutung zu [18].
Eine umfassendere evidenzbasierte Sichtweise findet sich in wissenschaftlich international abgestimmten Empfehlungen zur Prävention, Diagnose und Therapie der Osteoporose [19] bzw. für den deutschen Sprachraum z. B. im Weißbuch Osteoporose [20] oder in den Leitlinien des Dachverbandes der deutschsprachigen wissenschaftlichen Gesellschaften für Osteologie (DVO) für Osteoporose bei postmenopausalen Frauen und bei älteren Menschen [21]. Maßnahmen der Gesundheitsförderung im Sinne einer Ressourcenstärkung im jüngeren Lebensalter werden dort ebenfalls angesprochen. Hinsichtlich des Outcomes „Fragilitätsfraktur“ verdienen insbesondere Maßnahmen der Sturzprävention besondere Beachtung [22 - 24].

\section{Ethik und Verteilungsgerechtigkeit}

Grundsätzlich ist offen, auf Basis welcher ethischen Handlungsrichtlinien Entscheidungen getroffen werden sollten [25]. Die zur Auswahl stehenden ethischen Theorien führen in ihrer Konsequenz durchaus zu unterschiedlichen Ergebnissen. Nach dem Gleichheitsprinzip (Egalitarismus) könnte ein gleichmäßiger Ressourcenverbrauch oder ein gleichmäßiger Gesundheitszustand angestrebt werden, nach dem Differenzprinzip (egalitärer Liberalismus) eine vermehrte Ressourcenzuwendung zu den gesundheitlich Benachteiligten. Das Lohnprinzip würde auf die erbrachten Leistungen abstellen, das Vertragsprinzip auf getroffene Vereinbarungen, während das christliche Bedürftigkeitsprinzip ähnlich dem Differenzprinzip das „Not-wendige“ gewährt. Demgegenüber versucht das Nützlichkeitsprinzip (Utilitarismus), welches den dargelegten Kosten-Nutzwert-Analysen zugrunde liegt, den gesamtgesellschaftlichen Nutzen einer Handlungsstrategie angesichts prinzipiell beschränkter Ressourcen zu maximieren.

Kosteneffektivität ist auch kein absoluter oder abstrakt festzulegender Wert, sondern kontextuell definiert. Sie reflektiert die generelle Bereitschaft einer Gemeinschaft, begrenzte Ressourcen für gesundheitsbezogene Interventionen zu allozieren. Generell liegt derzeit die Grenze der Finanzierung für akutmedizinische Interventionen bei etwa 50-100 T€ pro gewonnenes Qualitätsadjustiertes Lebensjahr (QALY), für präventive Maßnahmen werden häufig niedrigere Grenzen angesetzt. Diese Werte sind jedoch nicht festgeschrieben und auch nicht festschreibbar, sondern reflektieren die Kosteneffektivität üblicher medizinischer Interventionen [26, 27]. Darüber hinaus beziehen sie sich auf die gesamtgesellschaftliche Perspektive. Werden auch indirekte Kosten berücksichtigt, wie dies einer gesamtgesellschaftlichen Perspektive entspricht, ist generell ein günstigeres Kosteneffektivitätsverhältnis zu erwarten als aus der gewählten Sicht der Kostenträger.

\section{Ausblick}

Die vorgestellte Evidenz aus eigenen epidemiologischen Studien unterstützt die prospektive Evaluation gezielter, kosteneffektiver Präventionsstrategien wie z.B. den Einsatz von Medikamenten oder auch der Knochendichtemessung zu Screening-Zwecken an selektierten Hochrisikogruppen zur Prävention osteoporotischer Fragilitätsfrakturen. Sie hat damit das Potenzial, Rationalität und Wirtschaftlichkeit im Gesundheitswesen zu unterstützen, wie dies in $§ 135$ SGB V gefordert ist. Eine konkrete Umsetzung hängt jedoch von der Entwicklung eines ausreichend 
belastbaren Instrumentes der Risikobestimmung ab, welches zu niedrigen Kosten eine vorbereitende Risikostratifizierung von Bevölkerungsgruppen erlaubt (National Institutes of Health 2000). Darüber hinaus wird von der International Osteoporosis Foundation und anderen empfohlen, die Ergebnisse standardisierter Knochendichtemessungen an der Hüfte als Frakturrisiko über einen definierten (10-Jahres-)Zeitraum ( „Hüftfraktur-Äquivalent“) auszudrücken [18, 28, 29]. Diese Forderung verlangt die wissenschaftlich begründete Entwicklung eines Instrumentes zur Abschätzung des künftigen Frakturrisikos unter Berücksichtigung der Knochendichte. Ein derartiges Instrument des RisikoAssessments ist prinzipiell aufgrund veröffentlichter Studien darstellbar, allerdings empfiehlt sich eine ausreichende Validierung vor einer flächendeckenden Einführung. Hierfür hat die Entwicklung computerunterstützter Instrumente des RisikoAssessments auf Basis vorhandener Evidenz hohes Potenzial.

Auch potente Verfahren wie die Kosten-Nutzwert-Analysen bzw. Kosteneffektivitätsanalysen, welche epidemiologische Daten rational bewerten, haben ihre Grenzen, die es genauer zu eruieren gilt. Ein weiterer Arbeitspunkt für die Zukunft ist daher die ethische Diskussion, welche die Chancen und Risiken derartiger Ansätze diskutiert.

\section{Danksagung}

Diese Studien wurden in Teilen unterstützt durch eine Förderung durch das Bundesministerium für Bildung, Forschung und Technologie unter dem Förderkennzeichen 01 EG 9404 (Bayerischer Forschungsverbund Public Health - Öffentliche Gesundheit).

Der Artikel nimmt besonderen Bezug auf folgende Beiträge dieses Sonderheftes von Das Gesundheitswesen: [30-33].

\section{Literatur}

${ }^{1}$ Lippuner K, von Overbeck J, Perrelet R et al. Incidence and direct medical costs of hospitalizations due to osteoporotic fractures in Switzerland. Osteoporos Int 1977; 7: 414-425

2 European Communities, European Fundation For Osteoporosis. Report on osteoporosis in the European Community - Action for prevention. Bruessel: European Commission, 1989

${ }^{3}$ Genant $\mathrm{H}$, Cooper C, Poor $\mathrm{G}$ et al. Interim report and recommendations of the World Health Organization Task-Force for Osteoporosis. Osteoporos Int 1999; 10: 259-264

${ }^{4}$ Felsenberg D, Wieland E, Hammermeister $C$ et al. Prevalence of vertebral spinal deformities in women and men in Germany. EVOS group in Germany. Med Klin 1998; 93 (Suppl 2): 31 - 34

${ }^{5}$ Wildner M. Die Epidemiologie der proximalen Femurfraktur und ihre sozialmedizinische Bedeutung. Habilitationsschrift. Ludwig-Maximilians-Universität München, 2001

${ }^{6}$ Meisinger C, Wildner M, Döring A et al. Validität und Reliabilität von Probandenangaben zu Frakturen. Soz Präventivmed 2000; 45: $203-207$

${ }^{7}$ Meisinger C, Wildner M, Stieber J et al. Epidemiologie der Extremitätenfrakturen. Ergebnisse des MONICA-Surveys 1994/95 in der Region Augsburg. Der Orthopäde 2002; 31: $92-99$
${ }^{8}$ Wildner M, Sangha O, Clark DE et al. Independent living after fractures in the elderly. Osteoporosis Int 2002; 13: 579-585

${ }^{9}$ Wildner M, Markuzzi A, Casper W et al. Disparitäten der Krankenhaus-Fatalität nach proximalen Femurfrakturen in der DDR. Soz Praeventiv Med 1998; 43: 80-89

${ }^{10}$ Wildner M, Fischer R, Brunner A. Development of a questionnaire for quantitative assessment in the field of health and human rights. Soc Sci Med 2002; 55: 1725-1744

${ }^{11}$ Graafmans WC, Ooms ME, Hofstee HMA et al. Falls in the elderly: a prospective study of risk factors and risk profiles. Am J Epidemiol 1996; 143: $1129-1136$

12 O'Neill TW, Varlow J, Reeve J et al. Fall frequency and incidence of distal forearm fracture in the UK. J Epidemiol Community Health 1995; 49: $597-598$

${ }^{13}$ Ware JE, Kosinski M, Keller SK. SF-36 physical and mental health summary scales: a user's manual. Boston, MA: The Health Institute, 1994

${ }^{14}$ Lühmann D, Kohlmann T, Lange S et al. Die Rolle der Osteodensitometrie im Rahmen der Primär-, Sekundär- und Tertiärprävention / Therapie der Osteoporose. Lübeck: Institut für Sozialmedizin, Medizinische Universität Lübeck, 1998

${ }^{15}$ Hailey D, Sampietro-Colom L, Marshall D et al. The effectiveness of bone density measurement and associated treatments for prevention of fractures: an international collaborative review. Int J Technol Assess Health Care 1998; 14: 237 - 255

${ }^{16} \mathrm{SBU}$. Bone density measurement - a systematic review. J Int Med 1997; 241: $1-60$

17 Cummings SR, Melton LJ 3rd. Epidemiology and outcomes of osteoporotic fractures. Lancet 2002; 359: 1761 - 1767

18 Kanis JA. Diagnosis of osteoporosis and assessment of fracture risk. Lancet 2002; 359: 2002

19 [Anonymous] . Osteoporosis prevention, diagnosis, and therapy. JAMA 2001; 285: $785-795$

${ }^{20}$ Berufsverband der Fachärzte für Orthopädie (BVO) et al. Weißbuch Osteoporose. Berlin: BVO, 2004

${ }^{21}$ www.lutherhaus.de/osteo/leitlinien-dvo/ (Zugriff am 30.11.2004).

22 Chang JT, Morton SC, Rubenstein LZ et al. Interventions for the prevention of falls in older adults: systematic review and meta-analysis of randomized clinical trials. BMJ 2004; 328: 680-683

${ }^{23}$ Robertson MC, Campbell AJ, Gardner MM et al. Preventing injuries in older people by preventing falls: a meta-analysis of individual-level data. J Am Geriatr Soc 2002; 50: 905 - 911

${ }^{24}$ American Geriatrics society, British Geriatric Society, and American Academy of Orthopedic Surgeons Panel on Falls Prevention. Guideline for the prevention of falls in older persons. J Am Geriatr Soc 2001; 49: $664-672$

${ }^{25}$ Roberts MJ, Reich MR. Ethical analysis in public health. Lancet 2002; 359: 1055-1059

${ }^{26}$ Gold MR, Siegel JE, Russell LB et al. Cost-effectiveness in health and medicine. New York: Oxford University Press, 1996

${ }^{27}$ Teng TO, Adams ME, Pliskin JS et al. Five-hundred life-saving interventions and their cost-effectiveness. Risk Analysis 1995; 15: 369-390

${ }^{28}$ Delmas P. Do we need to change the WHO Definition of Osteoporosis. Osteoporos Int 2000; 11: 189-191

${ }^{29}$ Kanis J, Gluer C. An update on the diagnosis and assessment of osteoporosis with densitometry. Osteoporos Int 2000; 11: 192 - 202

30 Löwel H, Döring A, Schneider A et al. The MONICA Augsburg surveys basis for prospective cohort studies. Gesundheitswesen 2005; 67 S1: $\mathrm{S} 13-\mathrm{S} 18$

${ }^{31}$ Holle R, Happich M, Löwel H et al. KORA - A research platform for population based health research. Gesundheitswesen 2005; 67 S1: S19-S25

32 Wichmann HE, Gieger C, Illig T et al. KORA-gen - Resource for population genetics, controls and a broad spectrum of disease phenotypes. Gesundheitswesen 2005; 67 S1: S26-S30

${ }^{33}$ Löwel H, Meisinger C, Heier M et al. The population-based Acute Myocardial Infarction (AMI) Registry of the MONICA/KORA study region of Augsburg. Gesundheitswesen 2005; 67 S1: S31 -S37 